\title{
Coroebus undatus (Coleoptera: Buprestidae) sur chêne liège dans le Sud-Est de la France : estimation des dégâts, relations entre ceux-ci et certains facteurs du milieu
}

\author{
P Du Merle, M Attié \\ INRA, unité de zoologie forestière, Avenue A Vivaldi, F-84000 Avignon, France
}

(Reçu le 18 février 1992; accepté le 26 juin 1992)

\begin{abstract}
Résumé - Dans 52 placettes de chêne liège, Quercus suber L, réparties entre Maures et Esterel, on a dénombré, sur 5-15 arbres dont le liège avait été récemment levé, les nombres d'intersections entre les galeries de $C$ undatus (Fabr) visibles à la surface des troncs et les 4 lignes verticales passant respectivement au milieu des faces nord, est, sud et ouest de ceux-ci. Pour chaque arbre, on a calculé un indice de dégâts $I A$, égal à la moyenne des 4 dénombrements relatifs au premier $1,50 \mathrm{~m}$ du tronc, mesuré à partir du sol, et pour chaque placette un indice de dégâts $I P$, égal à la moyenne des valeurs prises par IA. Le pourcentage endommagé $(\mathrm{A} \%)$ de surface de tronc d'un arbre était approximativement lié à $I A$ par la formule : $\mathrm{A} \%=0,4 I A$. $C$ undatus était présent partout, mais $I A$ et $I P$ variaient respectivement de 0 à 27,6 et de 1,2 à 14,8 selon les arbres et les placettes. La base des troncs était nettement moins endommagée que le reste de leur surface, tandis que leurs faces sud et ouest l'étaient légèrement plus que les 2 autres. À l'intérieur des placettes, les arbres étaient endommagés au même degré, en moyenne, quels que soient le diamètre et sans doute aussi le degré d'ombrage de leurs troncs. L'indice $I P$ n'était lié ni au diamètre moyen, ni, semble-t-il, au degré moyen d'ombrage des troncs. II tendait à varier en sens inverse de l'altitude et était en moyenne plus élevé sur les versants sud.
\end{abstract}

Coroebus undatus / Quercus suber / insecte sous-corticole / estimation dégâts / facteurs liés à l'arbre / facteurs du milieu

Summary - Coroebus undatus (Coleoptera: Buprestidae) on cork oak in southeastern France: an estimate of damage and its relationship to environmental factors. In 52 plots distributed throughout the cork oak stands (Quercus suber $L$ ) of the Maures-Esterel region in southeastern France (fig 1) 5-15 oaks were selected from those whose cork had been removed less than 1 year before. On such trees, galleries made by the late-instar larvae of the last generation of Coroebus undatus (Fabr) are clearly visible at the surface of the stripped part of the stem. On each tree, the 4 vertical lines running through the middle of the north, east, south and west sides of the stripped part of stem were divided into $50-\mathrm{cm}$ long sections from soil surface, and intersections between galleries and each of these segments of lines were counted. For each tree and each plot a damage index was calculated as follows: the damage index of a tree (IA) was the mean total number of inter- 
sections per stem side counted on the first basal $1.50 \mathrm{~m}$ of the stem, that of a plot (IP) was the mean value of IA. IA may be considered to be roughtly proportional to the percentage of damaged stem surface $(A \%)$, ie, the ratio, expressed as a percentage, between the total surface of the galleries and the surface of the stem; both variables are approximately linked by the relationship: $A \%=0.4 I A$. C undatus occurred everywhere in the investigated area, but IA and IP ranged from 0 to 27.6 and 1.2 to 14.8 respectively, according to the trees and the plots (figs 2,3 ). The first basal $0.50 \mathrm{~m}$ of stems was on average $\approx$ twice less damaged than the rest of their surface, while their south and west sides were slightly more damaged than the 2 others. Within plots, trees were equally damaged (same mean IA value) whatever the diameter and probably also the shade degree of their stems. Between-plot variation in IP was not explained by the mean diameter nor probably by the mean shade degree of stems. $I P$ value tended to vary inversely with elevation, and was higher on average on southern slopes.

Coroebus undatus / Quercus suber / inner-bark boring insect / damage assessment / tree factor/environmental factor

\section{INTRODUCTION}

L'aire naturelle du chêne liège, Quercus suber $L$, s'étend à travers le bassin occidental de la Méditerranée, le Maroc atlantique, le Portugal et le Sud-Ouest de la France. La superficie totale de la forêt de chêne liège serait de l'ordre de $2.10^{6}$ ha, dont près de la moitié dans la péninsule ibérique (Natividade, 1956; Normandin, 1980; Seigue, 1985). En France, d'après les données de l'Inventaire forestier national, les formations boisées à chêne liège dominant couvrent 108000 ha (Normandin, 1980), dont 40000 environ sont concentrés dans la région composée des massifs siliceux des Maures et de l'Esterel (DRAF PACA, 1988).

Le liège a fait, au siècle dernier et au début de ce siècle, la fortune de l'économie forestière méditerranéenne, et notamment celle du massif des Maures. Mais son exploitation est très exigeante en main-d'œuvre, facteur qui est à l'origine de la grave crise traversée depuis par cette production (Dugelay, 1952; Abric, 1975; Normandin, 1980). A la suite de la destruction d'une grande partie des peuplements de pin maritime, Pinus pinaster Ait, des Maures et de I'Esterel par la cochenille Matsucoccus feytaudi Ducasse (Schvester, 1981), le chêne liège, qui croissait sou- vent en sous-étage du conifère, est devenu le principal élément du manteau forestier de cette région. D'un autre côté, on s'accorde à reconnaître qu'une reprise des activités économiques de la forêt méditerranéenne contribuerait de façon importante à diminuer sa vulnérabilité au feu. Enfin, la balance commerciale «liège» de la France est en fort déficit. Ces diverses considérations ont amené, ces dernières années, les reponsables de la région administrative Provence-Alpes-Côte d'Azur (PACA) à engager une action en faveur de la relance de la subériculture, d'autant que celle-ci pourrait être, à terme, créatrice d'emplois.

La qualité du liège est 1 des 2 critères essentiels de sa valeur marchande, l'autre étant son épaisseur; ainsi le prix du $\mathrm{kg}$ de liège peut-il varier, selon son origine, de 1 à 10 (Seigue, 1985). Au nombre des facteurs de la qualité du liège figurent certains insectes déprédateurs, dont le coléoptère buprestide Coroebus undatus (Fabr), ou "ver du liège". Ce bupreste est depuis longtemps réputé commettre des dégâts importants dans les suberaies des Maures et de l'Esterel (Schaefer, 1949), mais il n'y avait jusqu'à ces derniers temps jamais été étudiè. À la demande du Conseil régional et des services forestiers de la région PACA, ces suberaies ont fait l'objet en 1991 d'une enquête, se fixant 3 objectifs : 
définir une méthode d'évaluation des dégâts de $C$ undatus, estimer ces dégâts, identifier certains des facteurs du milieu physique ou forestier qui influencent la multiplication de l'insecte.

On présentera ci-dessous les résultats de cette étude, après un rappel, tout d'abord de certaines caractéristiques du chêne liège et de sa sylviculture, puis de la biologie de son déprédateur.

\section{LE CHÊNE LIÈGE ET COROEBUS UNDATUS : RAPPEL DE CERTAINES CARACTÉRISTIQUES}

Les tissus composant le tronc et les branches des chênes lièges sont le résultat du fonctionnement de 2 assises génératrices concentriques : le cambium, qui produit du bois vers l'intérieur et du liber vers l'extérieur, l'assise subéro-phellodermique ou phellogène, qui produit de phelloderme vers l'intérieur et du liège vers l'extérieur. L'ensemble des tissus compris entre le bois et le liège constitue la "mère" du liège. On distingue 2 types de liège : le liège dit "mâle", qui est le liège originel de l'arbre, que ce dernier garderait toute sa vie si on ne l'enlevait pas, et le liège dit "femelle" ou "de reproduction", de bien meilleure qualité, produit par l'arbre après une récolte ou "levée» (Natividade, 1956; Seigue, 1985).

Dans la région Maures-Esterel, on préconise les règles d'exploitation suivantes du chêne liège : première levée, ou "démasclage», vers l'âge de 40 ans, lorsque la circonférence du tronc des arbres atteint environ $70 \mathrm{~cm}$; puis une levée tous les 12 ans environ du liège de reproduction (Vignes, 1990). Selon les caractéristiques des arbres, le liège est levé sur une portion seulement ou sur la totalité du tronc, parfois aussi à la base des grosses branches. L'époque des levées se situe en été.
Le bupreste $C$ undatus est distribué en Europe méridionale et moyenne (Portugal, Espagne, France, Italie, Suisse, Belgique, Allemagne, Autriche, Tchécoslovaquie, Hongrie, Yougoslavie, Roumanie) ainsi qu'en Afrique du Nord (Schaefer, 1949). II se développe à l'état larvaire aux dépens de nombreux chênes: Quercus suber, ilex $L$, mirbeckii Dur, pyrenaica Willd, lanuginosa Thuill, petraea (Matt) Liebl, robur L, peut-être aussi sur châtaigner, Castanea sativa Mill (Théry, 1942; Schaefer, 1949; Bachiller Bachiller et al, 1981).

Les adultes de $C$ undatus volent en mai-juin ou juin-juillet. Les œuis sont déposés dans les fissures de l'écorce. Les larves sont sous-corticoles. Sur chêne liège, elles creusent, au niveau du tronc et des grosses branches, entre la mère du liège et la couche subéreuse en formation, de longues galeries très sinueuses, dirigées dans tous les sens et qui pénètrent parfois brièvement dans l'aubier ou dans le liège. Ces galeries peuvent atteindre 1,80 $m$ de long et $6 \mathrm{~mm}$ de diamètre. Par suite de la production, par le phellogène, de nouvelles couches subéreuses, elles se trouvent assez rapidement incorporées dans l'épaisseur du liège. Une fois parvenues à maturité, les larves pénètrent dans le liège et y creusent une logette où elles se nymphosent. La durée du développement larvaire serait de 2 ans, parfois de 1 ou 3 ans (Perris, 1876; Barbey, 1925; Nonell I Comas, 1934; Schaefer, 1949; Wachtendort, 1955; Martin, 1961, 1964; Bachiller Bachiller et al, 1981).

Des dégâts importants de $C$ undatus ont été signalés au Portugal, en Espagne et en France sur chêne liège (Barbey, 1925; Nonell | Comas, 1934; Schaefer, 1949; Natividade, 1956; Martin, 1961, 1964; Bachiller Bachiller et al, 1981), en Allemagne du Sud sur Quercus robur et $Q$ petraea (Wachtenforf, 1955). Sur chêne liège, les dommages résultant de l'action du bupreste sont essentiellement de 2 
ordres : dépréciation de la qualité technologique du liège; risques d'arrachement de la mère lors de la levée du liège, ceci résultant de la formation, au niveau des zones où la mère a été lésée, d'adhérences entre le liège et elle (Natividade, 1956; Bachiller Bachiller et al, 1981).

On ne trouve dans la littérature à peu près aucune information précise sur les niveaux de population de $C$ undatus, sur la façon de les estimer, ni sur la dynamique des populations de l'insecte.

\section{LIEUX D'ÉTUDE, MATÉRIEL ET MÉTHODES}

\section{Choix des placettes d'étude}

Les observations sur le terrain ont été effectuées de mai à juillet 1991 dans des peuplements de chêne liège pur ou à chêne liège largement prédominant. En mai-juin, elles ont porté sur des arbres dont le liège avait été levé au cours de l'été précédent, en juillet sur des arbres dont le liège avait été levé quelques jours ou semaines seulement auparavant. Sur des arbres à liège levé depuis moins d'un an comme ceux étudiés ici, les galeries creusées par les larves de la dernière génération de $C$ undatus sont bien visibles à la surface de la partie déliégée du tronc. Lorsque la levée du liège est plus ancienne, l'appréciation des attaques du bupreste devient de plus en plus difficile, car les traces des galeries s'estompent progressivement avec le temps.

Les peuplements présentant des arbres dont le liège avait été récemment levé étaient peu nombreux, ce qui nous a empêché de multiplier le nombre des placettes autant que nous l'aurions souhaité. Au total 52 placettes, dont 39 levées en 1990 et 13 en 1991, ont pu être étudiées. Réparties entre Maures et Esterel et toutes situées dans le département du Var (fig 1), elles s'étageaient de 25 à $645 \mathrm{~m}$ d'altitude (voir plus loin, fig 3). L'altitude maximale de la région Maures-Esterel est de $780 \mathrm{~m}$ et le chêne liège n'y dépasse guère $700 \mathrm{~m}$.

Grasse

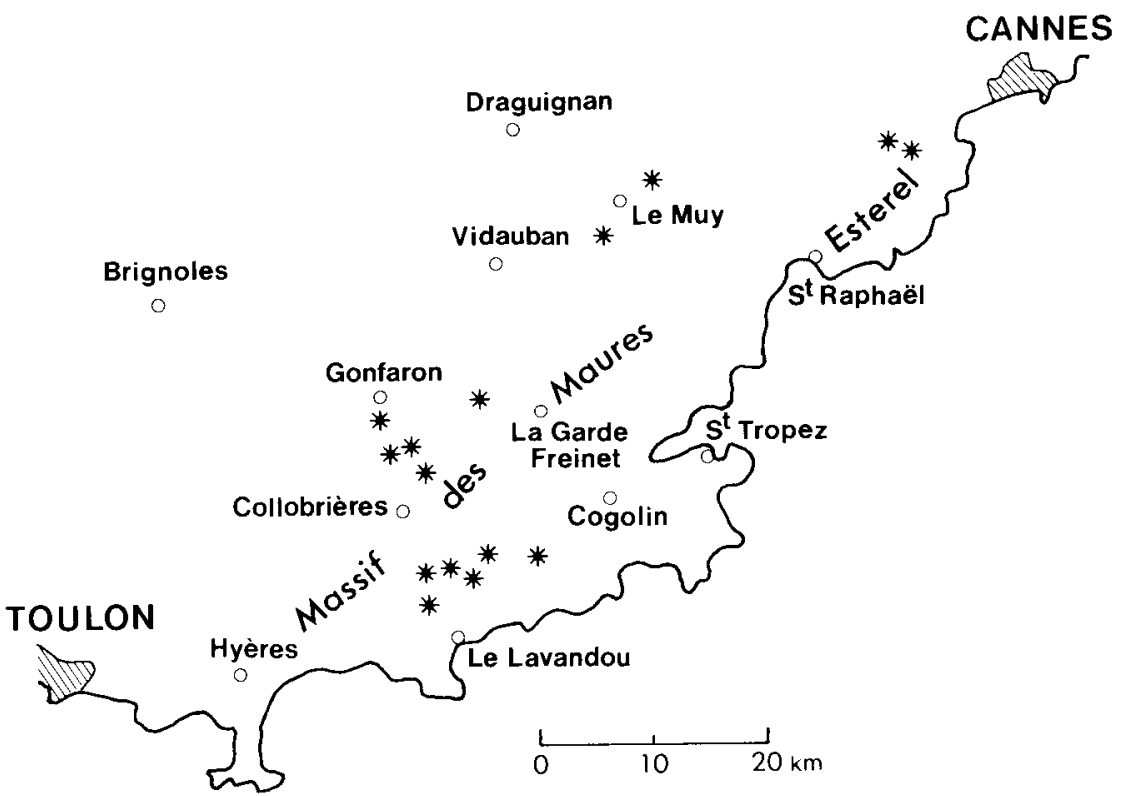

Fig 1. Situation géographique des lieux d'étude. Chaque étoile correspond à une ou plusieurs placettes. 
Pour chaque placette ont été notées les informations suivantes : altitude, exposition, pente, description sommaire de la végétation.

\section{Choix des arbres}

Selon la variabilité, appréciée visuellement, du diamètre des troncs dont le liège avait été levé, les observations ont porté sur 5 arbres ou sur un multiple de 5 arbres par placette. On a ainsi examiné :

- 5 arbres par placette dans 38 placettes où le diamètre des arbres variait assez faiblement;

- 10 arbres par placette, dont 5 pris parmi les plus petits et 5 parmi les plus gros, dans 12 placettes où ce diamètre variait fortement;

- 15 arbres par placette, dont 5 pris parmi les plus petits, 5 parmi les moyens et 5 parmi les plus gros, dans 2 placettes où ce diamètre variait également fortement.

Au total, 340 arbres ont été étudiés. Seuls ont été choisis des individus dont le liège avait été levé sur au moins $1,30 \mathrm{~m}$ de haut à partir du niveau de la surface du sol sur toutes les faces du tronc.

Pour chaque arbre ont été mesurés ou notés: la circonférence du tronc à $1,30 \mathrm{~m}$ $\left(C_{130}\right)$, toujours prise, par conséquent, sur la portion déliégée du tronc; la hauteur déliégée du tronc sur chacune de ses faces nord, est, sud et ouest; I' 'indice d'ombre" du tronc. Cet indice, d'autant plus élevé que le tronc était plus ombragé, a été défini d'après l'ampleur du houppier et la densité de son feuillage, d'après le degré de fermeture du peuplement au voisinage de l'arbre et d'après la densité et la hauteur de la végétation arbustive entourant celui-ci; il ne tenait pas compte des effets éventuels du relief. L'indice d'ombre a été noté selon une échelle à 6 classes, s'étendant de 0 (peuplement clairsemé à houppiers clairs ou réduits, végétation arbustive nulle aux alentours de l'arbre) à 5 (peuplement fermé à houppiers denses et/ou végétation arbustive épaisse et haute autour du tronc).

\section{Estimation de l'abondance des galeries}

Les galeries visibles à la surface des troncs dont le liège a été levé sont parfois disconti- nues, en raison de pénétrations temporaires des larves dans le liège ou l'aubier. Lorsque, de plus, les arbres ont été fortement attaqués, il devient difficile, voire impossible, de distinguer les galeries les unes des autres tant elles s'entrecroisent. Aussi, plutôt que de chercher à dénombrer les galeries présentées par les arbres échantilions, avons-nous procédé de la façon suivante. Sur chaque arbre, nous avons compté, sur chacune des faces nord, est, sud et ouest du tronc, le nombre de fois que la verticale passant au milieu de la face considérée était coupée par une galerie. Ces dénombrements ont été effectués séparément pour les premiers $50 \mathrm{~cm}$ du tronc à partir du sol $(=\mathrm{ni}-$ veau 1), puis pour les $50 \mathrm{~cm}$ suivants (= niveau 2), et ainsi de suite sur l'ensemble de la hauteur de tronc déliégée. Si le liège d'un arbre avait été, par exemple, levé sur une hauteur de 2,14 $\mathrm{m}$, cet arbre a donc fait l'objet de $4 \times 5=20$ dénombrements d'intersections, dont 4 relatifs à une portion de tronc (le niveau 5 , le plus haut) longue de seulement $14 \mathrm{~cm}$.

Les galeries creusées par les jeunes stades larvaires de $C$ undatus sont souvent difficiles à discerner ou sont devenues invisibles parce qu'ayant déjà été incorporées dans le liège levé. Seules, par suite, ont été prises en compte, lors des dénombrements d'intersections, celles des galeries dont le diamètre était au moins égal à $3 \mathrm{~mm}$ environ.

\section{Définition de l'indice de dégâts des arbres et des placettes}

Un indice de dégâts $I A$ a été calculé pour chacun des arbres étudiés. La hauteur déliégée des troncs variait fortement selon les arbres, de 1,30 à $2,22 \mathrm{~m}$; chez beaucoup, elle n'atteignait ou ne dépassait pas $1,50 \mathrm{~m}$. Seul le premier $1,50 \mathrm{~m}$ des troncs a par suite été pris en considération pour le calcul de l'indice de dégâts. $N, E, S$ et $O$ étant les valeurs respectives, pour chacune des faces nord, est, sud et ouest d'un tronc, de l'effectif cumulé des intersections observées sur les niveaux 1,2 et 3 de celui-ci, cet indice a été défini comme étant : $I A=(N+E+S+O) / 4$. Lorsque le niveau 3 de la partie déliégée du tronc n'atteignait pas $50 \mathrm{~cm}$ de long sur une ou plusieurs de ses faces, le ou les nombres d'intersections correspondants ont été corrigés en proportion de façon à les rapporter à cette lon- 
gueur. Le bien-fondé d'une telle correction sera discuté et justifié dans le chapitre Résultats et discussion. Les niveaux 3 , rappelons-le, étaient toujours longs d'au moins $30 \mathrm{~cm}$.

IA est donc le nombre moyen d'intersections par face du tronc pour le premier $1,50 \mathrm{~m}$ de celui-ci.

Un indice de dégâts $I P$, égal à la moyenne des différentes valeurs de $I A$ relatives aux arbres échantillons, a de même été calculé pour chaque placette. Dans le cas des placettes comportant 10 ou 15 arbres échantillons, 5 de ceux-ci seulement, choisis par tirage au sort, ont été pris en compte pour le calcul de $I P$, de façon à ce que cet indice se rapporte toujours au même nombre d'arbres par placette. Le bienfondé de la méthode utilisée pour choisir les 5 arbres sera lui aussi justifié dans le chapitre suivant.

\section{RÉSULTATS ET DISCUSSION}

\section{Signification des nombres d'intersec- tions et des indices de dégâts}

II ressort de nos observations sur le terrain que toutes ou quasiment toutes les galeries visibles à la surface des troncs avaient été creusées par des larves appartenant à la génération parvenue à l'état imaginal au cours de l'année de la levée du liège. Les dénombrements ici analysés se rapportaient donc à une seule génération de l'insecte, celle ayant achevé son développement larvaire en 1990 ou en 1991 selon la placette.

Soit $d$ le diamètre moyen d'une galerie de $C$ undatus et $\alpha$ son angle moyen avec l'horizontale, n'étant ici considérées que les grosses galeries du type de celles prises en compte dans les dénombrements. Considérons alors une portion de tronc de hauteur $h \mathrm{~mm}$ et soit $i$ le nombre d'intersections, pour cette portion de tronc, entre les galeries du bupreste et une certaine verticale. Si cette dernière, au lieu d'être une ligne sans épaisseur, était une bande très étroite, le nombre d'intersections entre les galeries et cette bande serait toujours $i$. Prenons la largeur de la bande égale à $1 \mathrm{~mm}$. Chaque intersection forme alors un parallélogramme de hauteur moyenne $d$ et de base moyenne $1 /$ $\cos \alpha$. À l'erreur statistique près, la surface de chaque intersection est égale à : $d /$ $\cos \alpha \mathrm{mm}^{2}$, et par suite celle cumulée des $i$ intersections à : $i d / \cos \alpha \mathrm{mm}^{2}$. La surface totale de la bande étant elle-même égale à $h \mathrm{~mm}^{2}$, le pourcentage endommagé de cette surface a donc pour estimation : 100 $i d / h \cos \alpha$.

Si $i_{m}$ est le nombre moyen des intersections entre les galeries et une verticale quelconque de la portion étudiée du tronc, il en découle que le pourcentage endommagé $A \%$ de la surface totale de cette portion a pour estimation :

$$
A \%=100 i_{\mathrm{m}} d / h \cos \alpha
$$

Supposons maintenant que $d$ et $\alpha$ ne varient pas entre les niveaux ou les faces des troncs et qu'ils ne sont pas influencés non plus par les caractéristiques des arbres et des peuplements. Sous cette hypothèse, $100 d / h \cos \alpha$ est constant pour $h$ donné. Les valeurs prises par $i_{m}$ sont alors, à ce coefficient constant près, des estimations de l'intensité, exprimée en pourcents endommagés de surface de tronc, des dégâts commis par la génération étudiée de $C$ undatus. En particulier, $I A$ et $I P$, qui sont des nombres moyens d'intersections et qui se rapportent à une valeur de $h$ égale à $1500 \mathrm{~mm}$, sont des estimations, au coefficient constant $d / 15$ $\cos \alpha$ près, de l'intensité des dégâts pour le premier $1,50 \mathrm{~m}$ des troncs.

L'hypothèse émise ci-dessus est très vraisemblable en ce qui concerne la relation entre $d$ (qui n'est autre que le diamètre moyen des grosses larves à l'origine des dégâts) et les caractéristiques des arbres et des peuplements, mais elie demande à 
être vérifiée pour le reste. En tout état de cause, $i, i_{m}, I A$ et $I P$ peuvent être en tout cas considérés comme au moins approximativement proportionnels à l'intensité des dégâts.

Prenons pour valeurs de $d$ et de $\alpha$ les valeurs centrales des distributions respectives des diamètres et des inclinaisons des galeries, soit $4,5 \mathrm{~mm}$ (puisque le diamètre des galeries prises en compte variait de 3 à $6 \mathrm{~mm}$ environ) et $45^{\circ}$ (puisque l'inclinaison de ces galeries variait de 0 à $90^{\circ}$ ). Dans ces conditions, on a : $d / 15 \cos \alpha=$ $4,5 / 15 \cos 45^{\circ}=0,42$. Le pourcentage endommagé de surface de tronc d'un arbre et sa valeur moyenne pour une placette peuvent être alors respectivement estimés, en se limitant au premier $1,50 \mathrm{~m}$ des troncs et aux seules grosses galeries visibles à la surface de ceux-ci, par:

$$
\begin{array}{ll}
A \%=0,4 I A & \text { pour un arbre } \\
P \%=0,4 I P & \text { pour une placette }
\end{array}
$$

Les valeurs ainsi calculées de $A \%$ et de $P \%$ ne sont évidemment que des approximations, peut-être grossières, mais elles présentent l'intérêt de fournir un ordre de grandeur de l'intensité des dégâts commis par l'insecte. Dans les faits, on peut avancer que $d$ et $\alpha$, qui sont, rappelons-le, des moyennes, sont très vraisemblablement compris entre 4 et $5 \mathrm{~mm}$ pour $d$ et entre 30 et $60^{\circ}$ pour $\alpha$, donc que le coefficient de proportionnalité intervenant dans les formules (2) et (3) est lui-même très vraisemblablement compris entre 0,31 et 0,67 .

II faut enfin noter que les résultats fournis par les formules (1) à (3) sous-estiment l'intensité des dégâts puisque ne sont prises en compte ni la portion la plus tine, large de moins de $3 \mathrm{~mm}$, des galeries ni les logettes de nymphose ni les galeries creusées directement dans le liège par l'insecte, d'abord avant la confection de ces logettes, puis pour émerger à l'air libre. Mais on peut admettre que les dégâts cor- respondants représentent une fraction à peu près constante des dégâts totaux. $\mathrm{La}$ sous-estimation est donc sans influence sur la signification de $i, i_{\mathrm{m}}, I A$ et $I P$, dont les valeurs restent approximativement proportionnelles à l'intensité totale des dégâts.

\section{Répartition de $\mathrm{C}$ undatus et intensité de ses dégâts dans les Maures et I'Esterel}

$C$ undatus est présent partout dans les Maures et I'Esterel. Des 340 arbres examinés, 10 seulement, soit $2,9 \%$, n'avaient pas été attaqués $(I A=0)$. Dans chacune des 52 placettes, des dégâts de l'insecte ont été observés sur au moins 3 des 5 arbres échantillons.

L'indice de dégâts $I A$ des arbres variait de 0 à 27,6 (fig 2A). Celui $I P$ des placettes variait de 1,2 à 14,8 (fig $2 \mathrm{~B}$; voir aussi, plus loin, la figure 3 ). La variation entre placettes de IP était hautement significa-

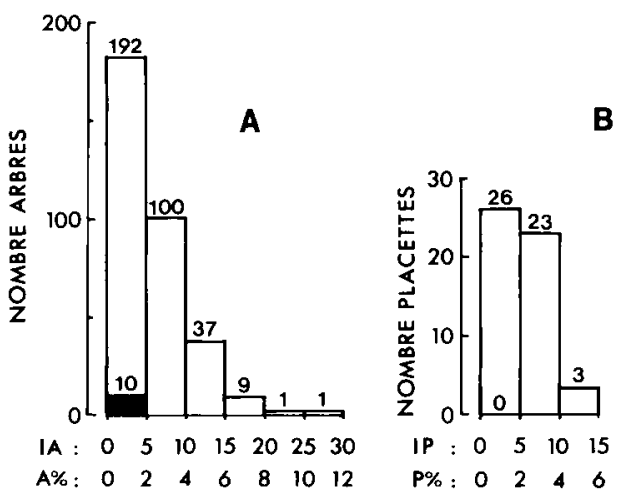

Fig 2. A : distribution des 340 arbres entre classes d'indice de dégâts (IA) ou classes de pourcentage endommagé estimé de surface de tronc $(A \%=0,4 / A)$. B : distribution des 52 placettes entre classes d'indice de dégâts $(I P=\Sigma$ $I A / 5)$ ou classes de pourcentage moyen endommagé estimé de surface de tronc $(P \%=0,4$ $I P$ ). Les fréquences des valeurs nulles de l'indice de dégâts sont indiquées (partie noire de la première colonne). 
tive (test de Kruskal-Wallis, $p=0,006$ ). La valeur moyenne de IP pour les 52 placettes était de 5,7. On peut en déduire, en appliquant les formules (2) et (3) soit directement soit après y avoir remplacé le coefficient de proportionnalité par les valeurs 0,31 et 0,67 entre lesquelles celui-ci est très vraisemblablement compris, que le pourcentage endommagé de surface de tronc variait de 0 à approximativement $11 \%(9-18 \%)$ selon les arbres, que la valeur moyenne par arbre de ce pourcentage variait approximativement de $0,5 \% \quad(0,4-$ $0,8 \%)$ à $6 \%(5-10 \%)$ selon les placettes et que cette valeur n'était que d'approximativement $2,3 \%(1,8-3,8 \%)$ pour une placette moyenne.

Au premier abord, il semblerait donc que $C$ undatus ne commette que des dégâts généralement modestes dans la région Maures-Esterel. II faut toutefois rappeler que les valeurs déduites des formules (2) et (3) sous-estiment l'importance des dégâts puisqu'elles ne prennent en compte que les grosses galeries. Les données recueillies, d'autre part, ne se rapportaient qu'à une seule génération de l'insecte, et il se peut que la densité de ce dernier varie largement selon l'année. Enfin, et surtout, il faut tenir compte des 2 facteurs suivants. Tout d'abord, un liège de qualité doit être dépourvu de défauts dans son épaisseur, notamment lorsqu'il est destiné à l'industrie du bouchon, débouché sans doute le plus intéressant de ce matériau au plan financier. La présence d'une galerie suffit par suite à déprécier fortement la valeur d'un volume de liège considérablement supérieur à celui de cette seule galerie puisqu'englobant toute l'épaisseur de la plaque de liège récoltée et s'étendant assez loin latéralement de part et d'autre de la galerie.

Le second facteur réside dans le fait que le liège produit par un arbre n'est réclté qu'à intervalles de temps de plusieurs années. Ce sont les dégâts cumulés com- mis par le bupreste entre deux levées successives du liège qu'il faut en fait prendre en compte. Selon Martin (1964), les chênes, après une récolte de liège, ne sont à nouveau attaqués par $C$ undatus qu'à partir de la $4^{e}$ année suivant celle de la levée. Puisque l'on recommande, dans la région Maures-Esterel, des levées espacées de 12 ans (Vignes, 1990), ce sont ainsi, si les indications de Martin sont exactes, les dégâts cumulés de $C$ undatus pendant 8 années consécutives environ qu'il faut considérer. En supposant que les pourcentages endommagés de surface de tronc estimés plus haut étaient voisins de leur valeur moyenne au cours des années précédentes, il faut donc multiplier par 8 ces pourcentages pour obtenir une estimation des dégâts de $C$ undatus présentés par le liège qui avait été récolté sur les arbres échantillons. En valeur cumulée, le pourcentage endommagé estimé de surface de tronc - c'est-à-dire, en négligeant d'éventuelles superpositions de galeries dans l'espace, le pourcentage estimé, en surface, du liège récolté présentant les restes d'une grosse galerie dans son épaisseur - variait alors de 0 à $88 \%$ (72$144 \%$ ) selon les arbres tandis que sa valeur moyenne par arbre variait de $4 \%$ (3$6 \%)$ à $48 \%(40-80 \%)$ selon la placette et s'élevait à $18 \%$ (14-30\%) pour une placette moyenne. En elles-mêmes, mais aussi compte tenu des considérations énoncées dans le paragraphe précédent, ces valeurs montrent clairement que la réputation d'insecte nuisible faite à $C$ undatus dans la région Maures-Esterel est loin d'être usurpée.

Dans cette région, l'intensité des dégâts de $C$ undatus et sa variance entre arbres dans les placettes variaient toutes 2 fortement d'une placette à l'autre. Selon la placette, IP variait de 1,2 à 14,8 , soit d'un facteur 12 , tandis que la variance estimée de IA variait de 0,85 à 64,90 , donc d'un facteur 76. Après transformation $\sqrt{ } \mathrm{x}$ ou $\log$ 
$(x+0,1)$ des valeurs de $I A$, cette variance variait encore respectivement de 0,06 à 2,40 et de 0,06 à 4,13 , donc d'un facteur 40 ou 69 . Ceci nous a conduits, lors des analyses des données présentées dans les chapitres suivants, à délaisser les méthodes statistiques classiques, notamment celles de l'analyse de variance, et à n'utiliser que des tests non-paramétriques.

\section{Distribution des dégâts à la surface des troncs}

\section{Selon les divers niveaux du tronc}

Les données analysées ont été celles relatives:

- a) aux placettes dans lesquelles le liège de chacun des 5 arbres échantillons avait été levé sur au moins $1,50 \mathrm{~m}$ de haut sur chacune des faces du tronc; ces placettes étaient au nombre de 31 ;

- b) à l'ensemble des 52 placettes (5 arbres/placette);

- c) à 31 placettes représentées chacune par 1 arbre dont le liège avait été levé sur au moins $2 \mathrm{~m}$ de hauteur sur chacune des faces du tronc; lorsque plusieurs arbres d'une placette présentaient cette caractéristique, un seul a été conservé pour les calculs après tirage au sort.

Les séries a) et b) ont servi à comparer les niveaux 1, 2 et 3 des troncs, la série c) à comparer les niveaux $1,2,3$ et 4 . Pour chacun des arbres et pour chacun des niveaux, on a calculé le nombre total des intersections observées sur l'ensemble des 4 faces du tronc, ceci après avoir, dans le cas de la série b), corrigé les données relatives aux niveaux 3 incomplets de telle sorte qu'elles se rapportent à une longueur de $50 \mathrm{~cm}$. Les analyses ont porté sur ces nombres d'intersections dans le cas de la série c), sur leurs moyennes par arbre pour chaque placette dans celui des séries a) et b). Les moyennes générales, pour l'ensemble des placettes, des nombres d'intersections par arbre sont présentées au tableau I. Les $31 \times 3$ et $52 \times 3$ moyennes par arbre et par placette calculées à partir des séries a) et b) de même que les $31 \times 4$ valeurs par arbre (= par placette) calculées à partir de la série c) ont été tout d'abord soumises à un test de

Tableau I. Moyennes générales, pour chaque niveau des troncs (= tronçon de $50 \mathrm{~cm}$ ), des nombres d'intersections par arbre (chaque arbre étant représenté par la somme de ses 4 faces), pour : A : 31 placettes où le liège de chacun des 5 arbres avait été levé sur au moins $1,50 \mathrm{~m}$ de haut sur chacune des faces du tronc, B : l'ensemble des 52 placettes, après correction des données relatives aux niveaux 3 incomplets, $C: 31$ placettes représentées chacune par 1 arbre dont le liège avait été levé sur au moins $2 \mathrm{~m}$ de haut sur chacune des faces du tronc. Chaque moyenne est suivie de son erreur standard, calculée à partir des moyennes par placette ou, pour la $3^{\theta}$ série, des valeurs absolues de la variable pour chaque placette. Les moyennes d'une même ligne suivies d'une même lettre ne diffèrent pas significativement $(p>0,05)$.

Origine des données

Niveaux du tronc

\begin{tabular}{ccrc}
\hline 1 & 2 & 3 & 4 \\
& & & \\
$5,2 \pm 0,6^{\mathrm{a}}$ & $9,7 \pm 0,8^{\mathrm{b}}$ & $10,2 \pm 0,7^{\mathrm{b}}$ & - \\
$4,8 \pm 0,4^{\mathrm{a}}$ & $9,0 \pm 0,7^{\mathrm{b}}$ & $9,2 \pm 0,6^{\mathrm{b}}$ & - \\
$4,4 \pm 0,6^{\mathrm{a}}$ & $8,4 \pm 1,0^{\mathrm{b}}$ & $9,3 \pm 1,2^{\mathrm{b}}$ & $8,1 \pm 1,1^{\mathrm{b}}$ \\
\hline
\end{tabular}
A : 31 placettes (5 arbres/placette)
B : 52 placettes (5 arbres/placette)
$4,8 \pm 0,4^{\mathrm{a}}$
$9,0 \pm 0,7^{\mathrm{b}}$
$9,2 \pm 0,6^{b}$
C : 31 placettes (1 arbre/placette) 
Friedman, qui a mis en évidence, pour chacune des 3 séries, un effet significatif du niveau du tronc $(p<0,001)$, puis au test non paramétrique de comparaisons multiples de moyennes pour échantillons non indépendants décrit par Noether (1976).

Les résultats de ces analyses sont parfaitement concordants (tableau 1). Le niveau 1 des troncs, c'est-à-dire leur premier $0,50 \mathrm{~m}$ à partir de la surface du sol, se distinguait significativement des niveaux 2,3 et 4 par un nombre moyen d'intersections près de 2 fois moindre et se montrait par conséquent près de 2 fois moins endommagé, en moyenne, par le bupreste. En revanche, les niveaux 2,3 et 4 ne différaient pas significativement entre eux sous ce rapport.

Le fait que les 3 jeux de données, et plus particulièrement les jeux a) et $b$ ), conduisent à la même conclusion en ce qui concerne la comparaison des niveaux 2 et 3 est important car il montre que la prise en compte, dans le cas du jeu b), de nombreuses données corrigées se rapportant à des niveaux 3 incomplets n'a pas eu d'effet sensible. Ceci se trouve confirmé lorsqu'on compare les seuls niveaux 3 incomplets aux niveaux 2 correspondants. Pour chacune des placettes, au nombre de 19, comprenant au moins un arbre dont le liège avait été levé sur au plus $1,35 \mathrm{~cm}$ de haut, on a choisi, éventuellement par tirage au sort, l'arbre dont le liège avait été levé sur la plus faible hauteur. Puis on a calculé, pour chacun des 19 arbres retenus, le nombre moyen d'intersections au $\mathrm{cm}$ par face du tronc, d'une part pour le niveau 2 et de l'autre pour la partie étudiée du niveau 3. Les moyennes générales de ces valeurs (resp 0,18 et 0,16 ) ne différaient pas significativement (test de Wilcoxon pour échantillons appariés; $p>0,05$ ).

Puisque ni les niveaux 3 complets ni la partie basale des niveaux 3 incomplets ne différaient des niveaux 2 par le nombre moyen d'intersections au cm, c'est donc que les parties basale et apicale des niveaux 3 ne différaient pas non plus entre elles à cet égard. On peut en conclure que le fait d'avoir corrigé les données relatives aux niveaux 3 incomplets en appliquant une simple règle de trois n'a pas induit de biais.

La variabilité entre placettes de la distribution des dégâts entre les niveaux 1 à 3 des troncs, d'une part, entre les seuls niveaux 2 et 3, d'autre part, a été étudiée en soumettant les données a), relatives aux placettes dont les 5 arbres échantillons avaient tous un niveau 3 complet, à des tests du $\chi^{2}$. Les valeurs analysées ont été celles prises, dans chacune des 31 placettes et pour chacun des 2 ou 3 niveaux des troncs, par le nombre total des intersections observées sur les 5 arbres, toutes faces confondues. La distribution des dégâts entre les niveaux 1 à 3 variait significativement entre les placettes $\left(\chi^{2}=94,97\right.$; ddl $=60 ; p=0,003)$. Le dénombrement des intersections sur un seul des 3 niveaux aurait donc conduit à une appréciation erronée de la variation entre placettes des dégâts subis par le premier $1,50 \mathrm{~m}$ des troncs. En revanche, la distribution des dégâts entre les niveaux 2 et 3 - donc sans doute également entre les niveaux 2 , 3 et 4 - ne variait pas significativement entre les placettes $\left(\chi^{2}=29,97\right.$; ddl $=30$; $p=0,47)$.

\section{Selon la face du tronc}

Les données analysées ont été celles relatives à l'ensemble des 52 placettes ( 5 arbres/placette). Pour chacun des arbres et pour chacune des 4 faces de son tronc, on a calculé le nombre total des intersections observées sur le premier $1,50 \mathrm{~m}$ de ce dernier (niveaux 1 à 3), ceci après avoir corrigé les données relatives aux niveaux 3 incomplets. Puis on a calculé, pour chaque placette puis pour l'ensemble des 52 
placettes, les moyennes par arbre, pour chaque face des troncs, des valeurs ainsi obtenues. Les $52 \times 4$ moyennes par placette ont été soumises tout d'abord à un test de Friedman, qui a mis en évidence un effet significatif de l'orientation $(p<$ 0,01 ), puis au même test non paramétrique de comparaisons multiples de moyennes que ci-dessus.

Les résultats de cette analyse sont présentés au tableau II. IIs indiquent que les faces sud et ouest des arbres étaient, en moyenne, légèrement plus endommagées que leurs faces nord et est, de façon non significative toutefois pour la face ouest. Lors de son étude d'une pullulation de $C$ undatus sur Quercus robur et sur $Q$ petraea en Allemagne du sud, Wachtendorf (1955) a examiné la position des galeries des très jeunes larves sur les troncs. II en déduit que le bupreste avait déposé ses œufs sur le côté sud de ces derniers et que ses larves s'étaient ensuite dispersées de tous côtés. Nous ne savons pas si $C$ undatus se comporte de la même façon sur chêne liège dans le contexte climatiquement très différent des Maures et de l'Esterel, mais ceci pourrait expliquer en partie nos propres résultats.

La variabilité entre placettes de la distribution des dégâts entre les 4 faces des troncs a été étudiée à partir des données relatives aux 52 placettes. Les valeurs prises, pour chacune des placettes et pour chacune des 4 orientations, par le nombre total des intersections observées sur le premier $1,50 \mathrm{~m}$ de l'ensemble des 5 arbres échantillons ont été soumises à un test du $\chi^{2}$. La distribution des dégâts entre les 4 faces des troncs ne variait pas significativement entre les placettes $\left(\chi^{2}=173,54\right.$; $\mathrm{ddl}=153 ; p=0,12$ ). Si ce résultat et le caractère presque négligeable, bien que significatif, de l'influence de l'orientation de la face du tronc s'avéraient généralisables à tous les peuplements de chêne liège, on pourrait donc, au prix d'une légère erreur, se limiter, pour estimer l'intensité des dégâts du bupreste, au dénombrement des intersections sur une seule face des troncs.

\section{Relations entre intensité des dégâts de C undatus et certaines caractéristiques des arbres et du milieu}

\section{Relations entre intensité des dégâts et taille, âge ou nombre de levées des arbres}

Chez les 340 arbres examinés, le $C_{130}$ mesuré sous écorce variait de 38 à $183 \mathrm{~cm}$ selon les individus. Le liège des moins gros des arbres avait été levé pour la première fois (démasclage). Certains de ces

Tableau II. Moyennes générales, pour chaque face des troncs, des nombres d'intersections par arbre (chaque arbre étant représenté par son premier $1,50 \mathrm{~m}=$ niveau $1+$ niveau $2+$ niveau 3 ) pour les 52 placettes, après correction des données relatives aux niveaux 3 incomplets. Chaque moyenne est suivie de son erreur standard, calculée à partir des 52 moyennes par placette. Les moyennes suivies d'une même lettre ne diffèrent pas significativement $(p>0,05)$.

Origine des données

Faces du tronc

$\begin{array}{llll}\text { Nord } & \text { Est } & \text { Ouest }\end{array}$


arbres étaient toutefois assez loin d'avoir atteint la valeur préconisée du $C_{130}$ sur écorce, soit $70 \mathrm{~cm}$ environ. L'individu de $38 \mathrm{~cm}$ n'avait ainsi, lorsque son liège a été levé, qu'un $C_{130}$ sur écorce d'environ 50 $\mathrm{cm}$ (valeur estimée à partir du $C_{130}$ sous écorce et de l'épaisseur du liège se trouvant à la base de la partie haute, non démasclée, du tronc).

Le tableau III compare, pour chacune des 14 placettes où ont été distinguées 2 ou 3 classes de $C_{130}$, représentées chacune par 5 arbres échantillons, les valeurs moyennes par arbre de $I A$ pour chacune de ces classes. Les différences entre classes de $C_{130}$ n'étaient significatives dans aucune placette (tests de Mann-Whitney ou de Kruskal-Wallis selon le nombre de classes; $p>0,05)$. Les moyennes générales relatives aux 2 modalités extrêmes (colonnes $A$ et $\mathrm{C}$ du tableau) étaient quasiment identiques et ne différaient pas non plus significativement entre elles (test de Wilcoxon pour échantillons appariés, appliqué aux 14 couples de moyennes; $p>0,05$ ).

À l'intérieur des placettes, l'indice de dégâts $I A$ des arbres et par suite le pourcentage endommagé $A \%$ de la surface des troncs n'étaient donc pas influencés par le $C_{130}$ de ces derniers, même là où celui-ci variait fortement. Ceci revient à dire que $C$ undatus, dans chaque placette, a exploité au même degré, en valeur relative, les différentes classes de $C_{130}$ représentées. On peut admettre que la surface unitaire d'une galerie du bupreste est à peu près

Tableau III. Relation entre indice de dégâts $I A$ des arbres et circonférences des troncs à $1,30 \mathrm{~m}$ $\left(C_{130}\right)$ pour les 14 placettes où ont été distinguées 2 ou 3 classes de $C_{130}$, représentées chacune par 5 arbres; $A$ et $C$ sont les 2 classes extrêmes. Chaque moyenne est suivie de son erreur standard; les erreurs standard des moyennes générales ont été calculées à paritr des 14 moyennes par placette. Les moyennes d'une même ligne suivies de la même lettre ne diffèrent pas significativement $(p>$ $0,05)$.

\begin{tabular}{|c|c|c|c|c|c|c|}
\hline \multirow[t]{2}{*}{$\begin{array}{c}N^{\circ} \\
\text { placette }\end{array}$} & \multicolumn{3}{|c|}{$\begin{array}{c}\mathrm{C}_{130} \text { des troncs } \\
\text { (valeurs extrêmes, en } \mathrm{cm} \text { ) }\end{array}$} & \multicolumn{3}{|c|}{$\begin{array}{c}\text { Valeur moyenne de } \mathrm{IA} \\
\text { par arbre }\end{array}$} \\
\hline & $A$ & $B$ & $c$ & $A$ & $B$ & $C$ \\
\hline 1 & $110-123$ & & $51-60$ & $9,0 \pm 1,9^{a}$ & & $15,8 \pm 1,8^{a}$ \\
\hline 3 & $101-112$ & & $66-78$ & $3,7 \pm 1,0^{\mathrm{a}}$ & & $3,0 \pm 0,7^{a}$ \\
\hline 4 & $124-138$ & & $68-74$ & $2,0 \pm 0,8^{a}$ & & $1,9 \pm 1,1^{a}$ \\
\hline 5 & $104-116$ & & $65-86$ & $5,8 \pm 1,2^{a}$ & & $4,7 \pm 1,4^{a}$ \\
\hline 6 & $101-118$ & & $62-87$ & $9,8 \pm 1,8^{a}$ & & $9,0 \pm 1,5^{a}$ \\
\hline 17 & $79-91$ & & $43-49$ & $3,4 \pm 1,2^{a}$ & & $3,4 \pm 1,9^{a}$ \\
\hline 20 & $112-130$ & & $86-94$ & $5,2 \pm 0,8^{a}$ & & $2,8 \pm 0,7^{a}$ \\
\hline 22 & $111-136$ & & $76-85$ & $3,2 \pm 0,5^{a}$ & & $3,3 \pm 1,2^{a}$ \\
\hline 27 & $110-166$ & & $38-59$ & $3,6 \pm 1,1^{a}$ & & $2,0 \pm 0,8^{a}$ \\
\hline 29 & $93-104$ & & $68-77$ & $5,3 \pm 0,9^{a}$ & & $4,8 \pm 1,0^{a}$ \\
\hline 36 & $120-130$ & & $62-66$ & $3,7 \pm 1,0^{a}$ & & $5,4 \pm 0,7^{a}$ \\
\hline 38 & $132-147$ & $95-101$ & $67-79$ & $5,5 \pm 0,7^{a}$ & $5,2 \pm 1,6^{a}$ & $2,9 \pm 1,0^{a}$ \\
\hline 39 & $95-100$ & & $65-74$ & $10,5 \pm 2,3^{a}$ & & $9,5 \pm 2,5^{a}$ \\
\hline 52 & $107-124$ & $74-83$ & $50-59$ & $3,3 \pm 0,4^{a}$ & $5,1 \pm 1,8^{a}$ & $6,4 \pm 1,7^{a}$ \\
\hline Moyenne générale & & & & $5,5 \pm 0,7^{a}$ & & $5,4 \pm 1,0^{a}$ \\
\hline
\end{tabular}


constante et, en particulier, qu'elle n'est pas influencée par les caractéristiques des arbres et des peuplements. Dans ces conditions, la surface cumulée des galeries d'un arbre, et par suite le produit de la valeur de $A \%$ relative à cet arbre par la surface du tronc, sont proportionnels à l'effectif des larves ayant attaqué l'arbre en question, et vice versa. Comme la surface d'un tronc est à peu près proportionnelle à son $C_{130}$ et que, d'autre part, $A \%$ ne variait pas entre classes de $C_{130}$, les effectifs de larves par arbre, dans chaque placette, étaient donc à peu près proportionnels, en moyenne, aux $C_{130}$ des troncs. II en allait vraisemblablement de même pour le nombre initial d'œufs par arbre. Le choix des arbres par les femelles pondeuses du bupreste avait donc été fortement influencé par le $C_{130}$ ou plutôt, beaucoup plus vraisemblablement, par le diamètre des troncs, ce qui suggère l'intervention d'un «effet silhouette», bien connu chez d'autres insectes.

Les résultats montrent en outre que les échantillons de 5 arbres tirés au sort parmi les 10 ou 15 arbres examinés dans chacune des 14 placettes où le $C_{130}$ des individus variait fortement étaient tout autant représentatifs de leurs placettes respectives que pouvaient l'être des leurs les échantillons de 5 arbres pris directement dans les 38 autres placettes. Dans le cas où $I A$ et $A \%$ se seraient montrés influencés par le $C_{130}$ des arbres, il aurait fallu, pour que chaun des 14 échantillons de 5 arbres soit représentatif, que chaque classe de $C_{130} \mathrm{y}$ soit représentée au prorata de sa fréquence relative dans la placette correspondante.

Le coefficient de corrélation entre IP et le $C_{130}$ moyen des 5 arbres échantillons a été calculé pour l'ensemble des 52 placettes mais aussi pour les seules 38 d'entre elles à taille des arbres peu variable. II était pratiquement nul et non significatif dans les 2 cas (respectivement $r=-$ $0,011, d d l=50, p>0,90$ et $r=0,058, d d l=$ $36, p=0,73)$, en dépit d'une forte variation entre placettes du $C_{130}$ moyen (valeurs extrêmes pour les 52 placettes : 48 et 147 $\mathrm{cm})$. Les nuages de points correspondants, non présentés ici, ne montraient pas de structure particulière. Aucun effet de la taille des arbres sur l'indice de dégâts des placettes n'était donc sensible.

$\mathrm{Au}$ total, la taille des arbres n'expliquait donc ni les variations de l'indice de dégâts à l'intérieur des placettes ni ses variations entre celles-ci. Le liège des plus jeunes des arbres ou placettes étudiés venait d'être levé pour la première fois tandis que celui des arbres ou placettes les plus âgés l'avait déjà été à plusieurs reprises auparavant, au moins 3 fois dans certains cas. L'âge des arbres et le nombre des levées précédemment subies par ceux-ci paraissent ainsi n'avoir aucune influence sur l'intensité des dégâts (à moins que les 2 facteurs n'agissent en sens inverse et que leurs effets se compensent alors presque exactement, ce qui est douteux). En particulier, il ne semble pas que les arbres non démasclés se montrent plus ou moins sensibles au bupreste que ceux revêtus de liège de reproduction.

\section{Relations entre intensité des dégâts et indice d'ombre des arbres ou densité des peuplements}

Les 340 arbres examinés se répartissaient en 110,155 et 75 individus à indice d'ombre respectivement égal à 0 ou 1 , à 2 ou 3 et à 4 ou 5 . Dans 26 des 52 placettes, l'écart entre valeurs extrêmes de l'indice d'ombre chez les 5,10 ou 15 arbres examinés était de 2 points tandis que dans 14 autres cet écart était de 3 ou 4 points (l'indice d'ombre variant, par exemple, de 0 à 3 ou de 2 à 5 selon les arbres). Pour chacune de ces placettes, au nombre total de 40 , on a pris l'arbre dont l'indice d'ombre était le plus faible et celui dont l'indice d'ombre était le plus élevé, après tirage au sort en cas d'ex-aequo. Le tableau IV compare, d'une part pour l'ensemble des 40 
Tableau IV. Valeurs moyennes de l'indice de dégâts $I A$ des 2 arbres à indice d'ombre respectivement le plus faible et le plus élevé de chaque placette, pour : $\mathrm{A}$ : les 40 placettes où les indices d'ombre des 2 arbres différaient de 2 à 4 points, $B$ : les 14 placettes où ces indices d'ombre différaient de 3 ou 4 points. Chaque moyenne est suivie de son erreur-standard. Les moyennes d'une même ligne suivies de la même lettre ne diffèrent pas significativement $(p>0,05)$.

\begin{tabular}{llr} 
Origine des données & $\begin{array}{c}\text { Moyenne générale de IA } \\
\text { pour les arbres } \\
\text { à indice d'ombre }\end{array}$ \\
\cline { 2 - 3 } & "faible» & "élevé» \\
\hline A : 40 placettes & $5,5 \pm 0,5^{\mathrm{a}}$ & $5,3 \pm 0,8^{\mathrm{a}}$ \\
B $: 14$ placettes & $5,2 \pm 0,7^{\mathrm{a}}$ & $4,0 \pm 0,8^{\mathrm{a}}$
\end{tabular}

placettes et d'autre part pour les seules 14 placettes à écart de 3-4 points, les valeurs moyennes de IA pour chacune des deux catégories d'arbres. Ces moyennes ne différaient significativement pour aucun des 2 groupes de placettes (test de Wilcoxon pour échantillons appariés; $p>0,05$ ). II faut toutefois remarquer que les échantillons de couples de données n'étaient pas homogènes, ce qui limite la signification de ce résultat. II pourrait en effet se faire, par exemple, que le bupreste ne réagisse pas de la même façon à un écart de 3 points entre indices d'ombre selon que cet écart correspond à des valeurs de l'indice respectivement égales à 0 et 3 ou à 2 et 5 .

L'estimation du rôle joué par l'indice d'ombre dans la variabilité entre placettes de $I P$ est rendue difficile à la fois par le caractère de variable qualitative de cet indice et par le fait que celui-ci variait parfois assez largement entre arbres d'une même placette. Dans 24 des 52 placettes (en ne considérant que les 5 arbres échantillons habituels par placette), l'indice d'ombre ne variait pas d'un arbre à l'autre ou bien ne variait que d'un point, prenant alors les valeurs $a$ et $a+1$. Pour chacune de ces 24 placettes, on a pris pour estimation de la valeur moyenne de l'indice d'ombre la valeur unique de cet indice dans le premier cas, $a+0,5$ dans le second. L'indice d'ombre moyen variait de 0 à 3,5 selon la placette. Le coefficient de corrélation entre $I P$ et cet indice était faible et non significatif $(r=0,165 ; \mathrm{ddl}=22 ; p=0,44)$.

Le coefficient de corrélation entre $I A$ et indice d'ombre calculé pour l'ensemble des 340 arbres était quant à lui presque nul $(r=0,042)$.

$\mathrm{Au}$ total, ces différentes analyses n'ont donc pas révélé d'effet sensible de l'indice d'ombre, susceptible d'expliquer une partie de la variabilité de l'intensité des dégâts dans ou entre les placettes. En Allemagne, en revanche, donc sous des climats nettement plus froids, Wachtendorf (1955) a constaté que les arbres dont le tronc était bien ensoleillé étaient les seuls à être fortement attaqués par $C$ undatus.

Le fait que l'indice d'ombre des arbres n'ait pas influencé, apparemment, la répartition entre arbres des dégâts du bupreste dans les placettes montre qu'il n'avait sans doute pas influencé non plus, auparavant, le choix des arbres par les femelles pondeuses. II pourrait y avoir contradiction entre cette conclusion et l'hypothèse de l'intervention d'un effet silhouette dans le choix des arbres par l'insecte car l'indice d'ombre tenait compte non seulement de diverses caractéristiques des arbres mais aussi de l'importance de la végétation arbustive, donc d'un facteur essentiel de la «visibilité» des troncs. Faute d'avoir noté avec précision la part de la végétation arbustive dans les valeurs prises par l'indice d'ombre, il n'est toutefois pas possible de se faire une opinion à cet égard.

Les résultats suggèrent que la densité des arbres, qui est un des facteurs déter- 
minant l'indice d'ombre, n'influencerait pas IP. Au nombre des interventions sylvicoles susceptibles d'améliorer la productivité des suberaies de la région Maures-Esterel et qui, par ailleurs, pourraient peut-être, en diminuant la compétition pour l'eau et la lumière, avoir pour effet d'enrayer les phénomènes de dépérissement observés ces dernières années dans ces suberaies, figurent des opérations d'éclaircie sélective et de régénération des peuplements. De telles interventions, qui auraient pour conséquence, dans un premier temps, de diminuer l'indice d'ombre des troncs, seraient donc apparemment sans effet important sur l'intensité des dégâts de $C$ undatus. Mais ceci demande confirmation.

\section{Relations entre intensité des dégâts et altitude ou exposition des placettes}

Le coefficient de corrélation entre IP et l'altitude, calculé pour les 52 placettes, était négatif et significatif bien que faible ( $r=-$ 0,$282 ; \mathrm{ddl}=50 ; p<0,05$ ). D'un autre côté, et toujours pour les 52 placettes, les valeurs moyennes respectives de $I P$ pour chacune des 4 classes habituelles d'exposition (tableau V) différaient significativement (test de Kruskal-Wallis; $p<0,01$ ).

Tableau V. Relation entre indice de dégâts IP des placettes et exposition pour l'ensemble des 52 placettes. Chaque moyenne est suivie de son erreur standard. Les moyennes suivies d'une même lettre ne diffèrent pas significativement $(p>0,05)$.

\begin{tabular}{|c|c|c|}
\hline Exposition & $\begin{array}{c}\text { Nombre } \\
\text { de placettes }\end{array}$ & $\begin{array}{c}\text { Valeur moyenne } \\
\text { de IP }\end{array}$ \\
\hline
\end{tabular}

\begin{tabular}{lrl} 
Nord & 16 & $5,5 \pm 0,8^{\mathrm{ab}}$ \\
Est & 7 & $4,7 \pm 1,0^{\mathrm{ab}}$ \\
Sud & 18 & $7,5 \pm 0,6^{\mathrm{b}}$ \\
Ouest & 11 & $4,0 \pm 0,4^{\mathrm{a}}$ \\
\hline
\end{tabular}

Elles étaient assez voisines pour les versants nord, est et ouest; les versants sud se distinguaient par une valeur moyenne de $I P$ sensiblement plus élevée; mais ils ne différait significativement que des versants ouest, ce qui est a priori surprenant (test non paramétrique de comparaisons multiples de moyennes pour échantillons indépendants d'effectifs inégaux, selon Noether, 1976, au seuil de 0,05).

Ces résultats suggèrent une influence négative de l'atitude et positive de l'exposition sud sur $C$ undatus, qui manifesterait par conséquent une certaine thermophilie. Afin de tester cette hypothèse, les placettes ont été réparties en catégories définies à la fois par l'altitude et par l'exposition. Quatre classes d'altitude ont été distinguées $(0-100,100-200,200-300$ et $300-700 \mathrm{~m}$ ) et 2 classes seulement d'exposition, l'exposition sud étant opposée aux expositions nord, est et ouest. Afin de tenir compte d'un éventuel effet «année», on a analysé séparément les données relatives aux seules placettes où le liège avait été levé en 1990 et celles relatives à l'ensemble des placettes. Les données de base sont présentées à la figure 3. La catégorie "0-100 m/exposition sud" n'a pas

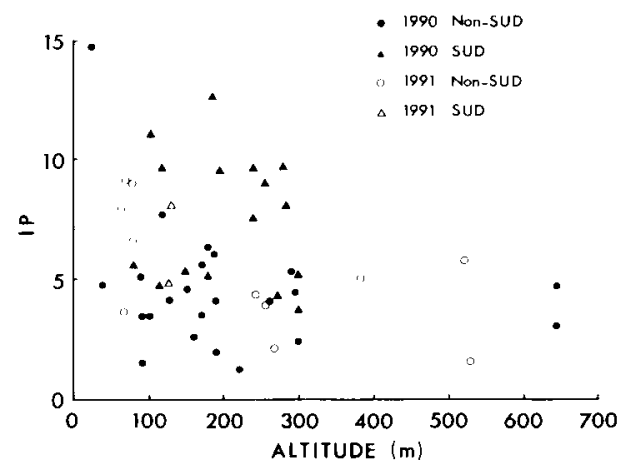

Fig 3. Relation entre indice de dégâts IP et altitude, selon l'année de levée du liège (1990 ou 1991) et l'exposition (sud ou "nond-sud»), pour les 52 placettes. 
été conservée car représentée par une seule placette. La catégorie $« 300-700 \mathrm{~m} /$ exposition sud" n'était quant à elle pas représentée du tout. L'analyse a donc porté finalement sur 38 et sur 51 placettes, chacun des 2 groupes étant réparti entre 6 catégories. Ses résultats figurent au tableau VI.

Pour chacune des 2 séries de placettes, les 6 catégories différaient significativement (test de Kruskal-Wallis; $p$ respectivement $<0,05$ et $<0,01$ ) et se classaient de la même façon. La valeur moyenne de IP était très nettement plus élevée aux expositions sud dans chacune des 2 tranches altitudinales (100-200 et 200-300 m) représentées pour les 2 classes d'expositions. Elle variait en sens inverse de l'altitude, mais assez faiblement, pour chacune des 2 classes d'exposition; sa valeur anormalement élevée pour la tranche altitudinale $300-700 \mathrm{~m}$ des expositions “non-sud» s'expliquait peutêtre par le très faible effectif des placettes, peut-être aussi par l'intervention d'un effet "année» ( 3 des 5 placettes, dans le cas de la seconde série de données, avaient été levées en 1991). Chacune des 2 séries de données a été soumise au test non paramétrique de comparaisons multiples de moyennes pour échantillons indépendants à effectifs inégaux proposé par Noether (1976). Les résultats de ces tests sont peu concluants (tableau VI), surtout dans le cas de la série des 38 placettes, pour laquelle il y a contradiction apparente avec le résultat du test de Kruskal-Wallis. Dans le cas étudié, qui se caractérisait tant par la forte variabilité de IP à l'intérieur de chaque catégorie de placettes (fig 3) que par les taibles effectifs de placettes (tableau VI), le test de Noether, qui est un test conservateur, a donc fait preuve d'un pouvoir de détection insuffisant.

Lorsqu'on compare les 2 classes d'exposition séparément pour chacune des 2 séries de données et pour chacune des tranches altitudinales $100-200 \mathrm{~m}$ et 200 $300 \mathrm{~m}$, ces 2 classes diffèrent toujours significativement entre elles (test de MannWhitney; $p<0,05$ ). En revanche, la comparaison, pour chacune des 2 séries de

Tableau VI. Relation entre indice de dégâts IP des placettes et altitude/exposition pour les placettes où le liège avait été levé en 1990 et pour l'ensemble des placettes. Deux classes d'exposition sont distinguées : "non-sud" ( $=$ nord + est + ouest) et sud. Les moyennes d'une même ligne suivies de la même lettre ne diffèrent pas significativement $(p>0,05)$. Dans le cas des placettes où le liège avait été levé en 1990, le test de comparaisons multiples de moyennes de Noether ne détecte aucune différence significative entre les moyennes alors que le test de Kruskal-Wallis indique que celles-ci diffèrent significativement (voir texte).

\begin{tabular}{|c|c|c|c|c|c|c|c|}
\hline & \multirow{2}{*}{$\begin{array}{l}\text { Altitude: } \\
\text { Exposition : }\end{array}$} & \multirow{2}{*}{$\begin{array}{l}0-100 \mathrm{~m} \\
\text { Non-Sud }\end{array}$} & \multicolumn{2}{|c|}{$100-200 \mathrm{~m}$} & \multicolumn{2}{|c|}{$200-300 \mathrm{~m}$} & \multirow{2}{*}{$\begin{array}{l}300-700 \mathrm{~m} \\
\text { Non-Sud }\end{array}$} \\
\hline & & & Sud & Non-Sud & Sud & Non-Sud & \\
\hline $\begin{array}{l}\text { Levée } \\
1990\end{array}$ & $\begin{array}{l}\text { Nbre de placettes } \\
\text { Moyenne IP } \\
\text { Erreur-standard }\end{array}$ & $\begin{array}{l}6 \\
5,6^{\mathrm{a}} \\
+1,9\end{array}$ & $\begin{aligned} & 7 \\
& 8,3^{a} \\
&+1,2\end{aligned}$ & $\begin{array}{c}10 \\
4,7^{a} \\
\pm 0,6\end{array}$ & $\begin{array}{l}8 \\
7,2^{\mathrm{a}} \\
\pm 0,9\end{array}$ & $\begin{array}{l}5 \\
3,5^{\mathrm{a}} \\
\pm 0,8\end{array}$ & $\begin{array}{l}2 \\
3,9^{a} \\
\pm 0,9\end{array}$ \\
\hline $\begin{array}{l}\text { Levées } \\
1990+1991\end{array}$ & $\begin{array}{l}\text { Nbre placettes } \\
\text { Moyenne IP } \\
\text { Erreur-standard }\end{array}$ & $\begin{array}{l}11 \\
6,4^{\mathrm{ab}} \\
\pm 1,1\end{array}$ & $\begin{array}{l}9 \\
7,9 a \\
+1,0\end{array}$ & $\begin{array}{l}10 \\
4,7^{\mathrm{ab}} \\
\pm 0,6\end{array}$ & $\begin{array}{l}8 \\
7,2^{\text {ab }} \\
\pm 0,9\end{array}$ & $\begin{array}{l}8 \\
3,5^{\mathrm{b}} \\
\pm 0,5\end{array}$ & $\begin{array}{l}5 \\
4,0^{\mathrm{ab}} \\
\pm 0,8\end{array}$ \\
\hline
\end{tabular}


données et pour chacune des 2 classes d'exposition, des différentes tranches altitudinales ne met en évidence aucune différence significative entre celles-ci (tests de Mann-Whitney ou de Kruskal-Wallis; $p>$ $0,05)$.

Finalement, et bien que la différence apparente de sensibilité de $C$ undatus à l'altitude et à l'exposition soit assez surprenante, l'hypothèse d'une certaine thermophilie de bupreste, se traduisant par des dégâts en moyenne plus importants aux basses altitudes et plus encore aux expositions sud, semble pouvoir être retenue. En tout état de cause, cependant, ce facteur n'expliquait qu'une faible part de la très forte variabilité de $I P$ (fig 3). C'est ainsi, par exemple, que l'une des plus faibles valeurs de $I P$ et que sa plus forte valeur (respectivement 1,6 et $14,8)$ ont toutes 2 été enregistrées dans des placettes à la fois situées dans la tranche altitudinale 0-100 $\mathrm{m}$ et exposées au nord.

\section{Influence d'autres facteurs du milieu}

Le calcul, pour les 52 placettes, du coefficient de corrélation entre IP et la pente n'a pas révélé de liaison entre les 2 variables ( $r=-0,172 ; \mathrm{ddl}=50 ; p=0,27$ ).

En Catalogne, selon Nonell I Comas (1934), C undatus préfère les milieux secs. Wachtendorf (1955), d'un autre côté, attribue les pullulations du bupreste observées peu après la seconde guerre mondiale sur Quercus robur et sur $Q$ petraea en Allemagne du Sud - donc dans un contexte bioclimatique très différent de celui des suberaies de la région MauresEsterel - à un affaiblissement des arbres résultant d'une alimentation en eau défavorable. L'examen des relations entre les valeurs prises par IP dans nos placettes et le caractère plus ou moins sec ou hu- mide de ces dernières, apprécié intuitivement sur le terrain ou par le biais de l'abondance de certaines espèces végétales comme la fougère Pteridium aquilinum $(L)$ Kuhn, n'a pas fait ressortir de liaison entre les 2 variables.

\section{CONCLUSIONS}

La méthode décrite dans cet article permet de quantifier avec une certaine précision, qu'il resterait à mieux définir, l'ampleur des dégâts de $C$ undatus sur chêne liège. Sa principale limite réside dans le fait qu'elle ne peut s'appliquer qu'à des arbres dont le liège a été récemment levé et qu'à la seule génération de l'insecte parvenue à l'état imaginal l'année de la levée. $C$ undatus se montrait présent partout dans la région Maures-Esterel, où ses dégâts, d'intensité très variable selon l'endroit, atteignaient parfois des niveaux pouvant être qualifiés d'élevés. La base des troncs était nettement moins endommagée par l'insecte que le reste de ceux$\mathrm{ci}$, tandis que leurs faces sud et ouest l'étaient légèrement plus que les 2 autres. À l'intérieur des placettes, les arbres étaient endommagés, en moyenne, au même degré quels que soient le diamètre et sans doute aussi l'indice d'ombre de leurs troncs. L'indice de dégâts des placettes n'était lié ni au diamètre moyen ni, semble-t-il, à l'indice d'ombre moyen des troncs. En revanche, il tendait à varier en sens inverse de l'altitude et se montrait plus élevé, en moyenne, sur les versants sud, l'influence de ces deux facteurs restant toutefois à confirmer et à préciser. Sur le plan pratique, malheureusement, l'étude n'a pas fourni de résultats qui permettraient de proposer aux gestionnaires forestiers des mesures propres à limiter l'importance des dégâts commis par le bupreste. On notera cependant un résultat intéressant : il ne semble pas que les opé- 
rations d'éclaircie ou de régénération des peuplements puissent avoir pour effet de favoriser la multiplication de l'insecte.

\section{REMERCIEMENTS}

Cette étude a été réalisée avec le concours financier du Conseil régional de la région PACA. Nous tenons à remercier les Services forestiers régionaux (ONF et CRPF) pour leurs informations sur les suberaies des Maures et de l'Esterel, ainsi que $\mathrm{JL}$ Clément (CNRS-Marseille), G Bonin (Université Aix-Marseille III) et $H$ Oswald (INRA-Avignon) pour leurs conseils lors de la définition du protocole d'échantillonnage et lors de l'analyse des résultats.

\section{RÉFÉRENCES}

Abric EF (1975) Évolution de l'industrie du liège en Provence. Rev For Fr 27, 453-461

Bachiller Bachiller $P$, Cadahia Cicuéndez D, Ceballos Fernandez de Cordoba $G$ et al (1981) Plagas de insectos en las masas forestales españolas. Ministerio de Agricultura, Pesca y Alimentacion, Madrid

Barbey A (1925) Traité d'entomologie forestière à l'usage des sylviculteurs, des reboiseurs, des propriétaires de bois et des biologistes. $2^{\mathrm{e}}$ édition, Berger-Levrault, Paris

DRAF PACA (1988) Enquête annuelle de branche. Production forestière \& scierie. Résultats provisoires. Année 1987. Direction régionale de l'Agriculture et de la forêt Provence-Alpes-Côte d'Azur, Marseille
Dugelay A (1952) La production et l'industrie du liège en Provence. Rev For Fr 4, 728-751

Martin C (1961) Ensayos de tratamientos contra el Coraebus undatus Fabr y Mars. Bol Serv Plagas For 5 (8), 73-82

Martin C (1964) Notas sobre el Coraebus undatus Fabr y Mars, plaga del alcornoque. Bol Serv Plagas For 7 (14), 95-101

Natividade J Vieira (1956) Subériculture. École nationale des Eaux et Forêts, Nancy

Noether GE (1976) Introduction to statistics. A nonparametric approach. $2^{\theta}$ édition, Houghton Mifflin Co, Boston

Nonell I, Comas J (1934) Investigaciones biològiques sobre el corc del suro Coroebus undatus (Fabr). Mem $R$ Acad Cienc Nat Artes Barcelona 23, 481-497

Normandin $D(1980)$ L'économie du liège en France. Rev For Fr 32, 79-90

Perris É (1876) Larves de Coléoptères. Ann Soc Linn Lyon (NS) 22, 259-418

Schaefer L (1949) Les Buprestidae de France. Éd sci cabinet entomol E, Le Moult, Paris

Schvester D (1981) Pin maritime et Matsucoccus feytaudi Duc. État actuel de la question. For Méditerr 3, 149-153

Seigue A (1985) La forêt circumméditerranéenne et ses problèmes. Maisonneuve et Larose, Paris

Théry A (1942) Coléoptères Buprestides. Faune de France 41, Office central de Faunistique, Paris

Vignes É (1990) Sylviculture des suberaies varoises. For Méditerr 12, 125-130

Wachtendorf W (1955) Beiträge zur Kenntnis der Eichenprachtkäfer Agrilus biguttatus Fabr und Coraebus undatus Fabr (Col Bupr). $Z$ Angew Entomol 37, 327-339 